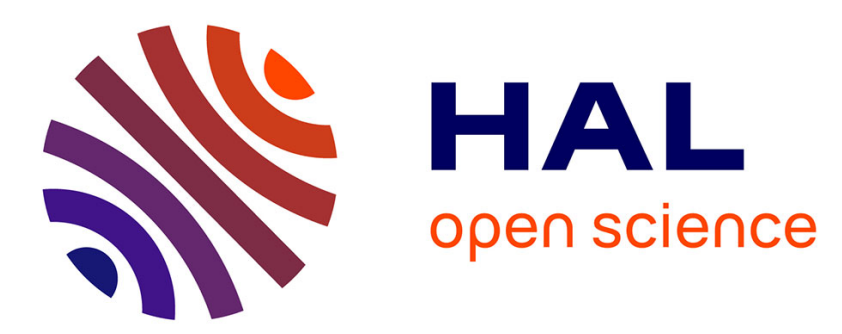

\title{
1D manipulation of a micrometer size particle actuated via thermocapillary convective flows
}

Ronald Terrazas, Aude Bolopion, Jean Beugnot, Pierre Lambert, Michaël Gauthier

\section{- To cite this version:}

Ronald Terrazas, Aude Bolopion, Jean Beugnot, Pierre Lambert, Michaël Gauthier. 1D manipulation of a micrometer size particle actuated via thermocapillary convective flows. RSJ International Conference on Intelligent Robots and Systems, Sep 2017, Vancouveur, Canada. hal-02300626

\section{HAL Id: hal-02300626 \\ https://hal.science/hal-02300626}

Submitted on 29 Sep 2019

HAL is a multi-disciplinary open access archive for the deposit and dissemination of scientific research documents, whether they are published or not. The documents may come from teaching and research institutions in France or abroad, or from public or private research centers.
L'archive ouverte pluridisciplinaire HAL, est destinée au dépôt et à la diffusion de documents scientifiques de niveau recherche, publiés ou non, émanant des établissements d'enseignement et de recherche français ou étrangers, des laboratoires publics ou privés. 


\title{
1D manipulation of a micrometer size particle actuated via thermocapillary convective flows
}

\author{
Ronald Terrazas Mallea ${ }^{1,2}$, Aude Bolopion ${ }^{2}$, Jean-Charles Beugnot ${ }^{2}$, Pierre Lambert $^{1}$ and Michael Gauthier ${ }^{2}$
}

\begin{abstract}
This paper deals with the open-loop characterization of a micromanipulation system actuated by thermocapillary convective flows. Micrometric size objects placed at the air/liquid interface are actuated by heating the surface of the liquid using a laser. The heat generates a surface tension gradient at the interface which induces thermocapillary convective flows that are used to move the objects. In this paper, the performances of this approach are analyzed based on openloop experiments. Several actuation strategies are proposed and discussed. The experimental results highlight the potential of this approach since velocities up to several millimeters per second are obtained. However the precision of the positioning is not ensured by open-loop actuation, so closed-loop control will be necessary in future works. As a first step towards closedloop control, this paper proposes a model of the system. This model is based on the open-loop experimental results, but the proposed methodology can be applied to any setup that use thermocapillary convective flows for particle manipulation.
\end{abstract}

\section{INTRODUCTION}

The goal of microrobotics is to develop fabrication, characterization and actuation techniques dedicated to objects whose size is below $1 \mathrm{~mm}$. This scale range includes objects of various natures, from electronic components to biological cells. The application domains are numerous and consequently, this research field has gained increasing interest over the last years. Non-contact actuation techniques are particularly attractive due to the versatility of use and the fact that they do not require the end effector to be in direct contact with the objects to be manipulated.

In this framework, dielectrophoresis uses a time variant electric field to apply a force to dielectric objects [1]. The electric field is produced by electrodes commonly fabricated by sputtering gold on a substrate. Objects in the range of 1 to $100 \mu \mathrm{m}$ have been controlled with velocities of several millimeters per second [2]. Depending on the properties of the objects and the medium, as well as on the electric field applied, the objects can be either attracted towards the electrodes (unstable situation) or repulsed from them (stable situation). The independent control of several objects can be realized but it needs specific control laws [3]. The main drawback of dielectrophoresis is that the electric field decays rapidly far from the electrodes. The size of the workspace is thus reduced or it needs complex

\footnotetext{
${ }^{1}$ Bio-, Electro- And Mechanical Systems department (BEAMS), Ecole Polytechnique de Bruxelles, Universite Libre de Bruxelles, 1050 Bruxelles, Belgium. rterraza@ulb.ac.be

2 FEMTO-ST Institute, AS2M and Optics departments, Univ. Bourgogne Franche-Comté, CNRS, Besancon, France. aude.bolopionefemto-st.fr
}

wire bonding methods to power a large number of electrodes.

Magnetophoresis is based on magnetic fields produced by electromagnetic coils [4] or moving permanent magnets [5]. The scalability of this technique is remarkable since objects of any shape as small as a few micrometers to objects larger than $1 \mathrm{~mm}$ can be controlled [6]. Several methods can be used to produce the desired displacement. Magnetotactic bacteria are self-propelled natural microrobots. The magnetic fields are used only to control the direction of the movement [7]. Alternatively, the magnetic field can produce a force [8] or a torque [9] on the object, such that both the position and the orientation can be controlled. However, as the magnetic and ferromagnetic objects are attracted towards high magnetic gradients areas (such as the coils), closed-loop control must be implemented to maintain the objects at a desired position. If several objects have to be controlled simultaneously but independently, strategies based on the nonlinearity of the magnetic field, or on the geometrical properties of the objects [10] must be used. Thanks to the large range of the magnetic fields, in vivo biomedical applications are foreseen [11]. Magnetophoresis is thus a highly promising technique due to its versatility. Its main limitation is that objects must have magnetic properties.

Lasers are also attractive tools. Two different properties are exploited by microrobotic systems: the generated optical forces and the generated heat.

Optical tweezers are based on the optical forces produced on dielectric objects by focalized lasers [12]. Single objects, as well as groups of objects can be controlled [13]. This actuation technique is demonstrated on both artificial and biological objects. If the objects cannot tolerate the temperature increase due to the laser beam, or if their properties do not suit optical tweezer manipulation requirements, indirect manipulation using intermediate tools controlled by laser trapping has been demonstrated [14]. Characterization of biological properties is possible using this tool. In [15], optical tweezers are used to get information about the mechanisms of chemoattractant gradient induction of cell migration.

Heat produced by the laser can be exploited as a fabrication tool or as an actuator. For example it can be used as a bonding tool to assemble micrometric size components in-situ [16]. Lasers are also valuable tools in the field of molecular biology. Microdissection can be performed either due to the melting effect or to the high photon density [17]. Regarding the use of lasers as actuators, the opening and closing of microgrippers has been achieved. In that 
case, the thermal expansion of the gripper arms due to the heat generated by the laser induces the open/close movement [18]. As another example, the heat generated by a laser focalized on a container filled with liquid can onset thermal-driven convective flows used to transport objects. These convective flows can be classified into two categories, depending if the liquid is heated from below, which induces natural and Marangoni convection, or from the top, which induces thermocapillary convection.

A combination of Marangoni and natural convective flows has been used to manipulate micrometer glass beads [19]. Selective manipulation is demonstrated by controlling the convective cell growth so that it affects only the beads that should be manipulated.

Alternatively, the liquid can be heated from the top which generates thermocapillary convection. This type of convection does not require a temperature gradient threshold and any temperature increase will onset the flow [20]. In addition, natural convection will not be generated. This makes the identification of the driving force easier as the only force driving the particles movement will come from the thermocapillary convective flow [21]. Details about the physics involved in thermocapillary convection are given in our previous work [21]. Several works use thermocapillary convection for particle manipulation. In [22], the thermocapillary convective flow is generated by heating a bubble interface to manipulate different types of particles such as glass and hydrogel beads or SU-8 triangular pieces. A slightly different approach was proposed in [23], where the bubble is generated by strongly heating the bottom of the fluid.

In this paper, we focus on the analysis of the performances of thermocapillary convection and on providing a model for this system that can be used for controlled micromanipulation. Two different actuation strategies are compared: one where the laser position is fixed and one where it follows the particle. Experimental tests are performed from which the attained velocities and the repeatability of the results are discussed. To ensure the positioning precision, a closed-loop control should be implemented in future works. To do so, a model of the system that relates the particle velocity to the laser position is proposed in this paper. Although the numerical values are related to the setup used in this work, the methodology can be applied to other setups that use thermocapillary convective flows. The relevance of this actuation principle compared to other classical micromanipulation techniques, such as magnetophoresis and dielectrophoresis is also discussed.

This paper is organized as follows. A short physical description and the setup details are provided in Sec. II. Two different actuation strategies are compared. A model of the system is proposed in Sec. III. Sec. IV discusses the relevance of this actuation technique in the framework of micromanipulation. Sec. V concludes the paper.

\section{THERMOCAPILLARY CONVECTIVE FLOW BASED MICROMANIPULATION}

\section{A. Physical description of thermocapillary convective flows}

A short description of the physics involved in thermocapillary convective flow based micromanipulation is provided here. More details can be found in [21]. The particles to be manipulated are placed at the air/liquid interface of a container filled with water. A laser beam coming from the top reaches the surface of the water. According to BeerLambert's law, part of this energy is absorbed by the liquid as it passes through. This absorbed energy is converted to heat and propagates in the fluid. This phenomenon is modeled by the heat equation which defines the temperature field in the entire fluid. Then, the temperature gradient at the interface generates a surface tension stress at the interface, which induces a movement of the fluid following the Navier-Stokes' equations, that define the velocity field across the entire fluid. The particles on the surface are dragged by the fluid.

\section{B. Experimental setup}

The setup used for thermocapillary convective flow based micromanipulation is presented in Fig. 1. The water container is placed on an anti-vibration table Newport M-IG-12-2. Spherical micrometer size particles (stainless steel AISI 304 Redhill Precision - diameter of $500 \mu \mathrm{m}$ ) are manually placed at the surface of the liquid (distilled water) contained in a plastic container $(115 \times 115 \mathrm{~mm})$. A plastic lid is put on top of the container to reduce dust deposition. The surface of the liquid is locally heated using a continuous-wave 1455 $\mathrm{nm}$ infrared laser (Keopsys Fiber Raman Laser). Since the infrared laser is not in the visible spectrum, it is coupled to a continuous-wave $655 \mathrm{~nm}$ red laser using a laser coupler so that the position of the laser spot and the one of the particle can be recorded by a Photonfocus camera (MVD1024-TrackCam) with a resolution of $1024 \times 1024$ pixels at a frame rate of 30 FPS. A $50 \mathrm{~mm}$ focal length lens is used together with a $10 \mathrm{~mm}$ extensor ring. The two laser beams are collimated using a 10x Olympus objective. The position of the laser spots are controlled using a 2-DOF piezoactuated tip/tilt mirror (Physik Instrumente S.334-2SL). The mirror is controlled via a PI Controller E-616.SS0x, which controls the mirror deviation. More details about the setup can be found in [21].

\section{Open-loop characterization of thermocapillary microma- nipulation}

To characterize the performances of the thermocapillary convective flow system for the manipulation of micrometric size objects, open-loop experiments are performed using two different strategies. On both cases, the particle velocity is analyzed.

The first strategy consists in positioning the laser $\mathbf{P}_{\text {las }}$ one millimeter away from the particle position, and keeping the laser position fixed during all the experiment. Three different samples of water are used, and for each sample, 

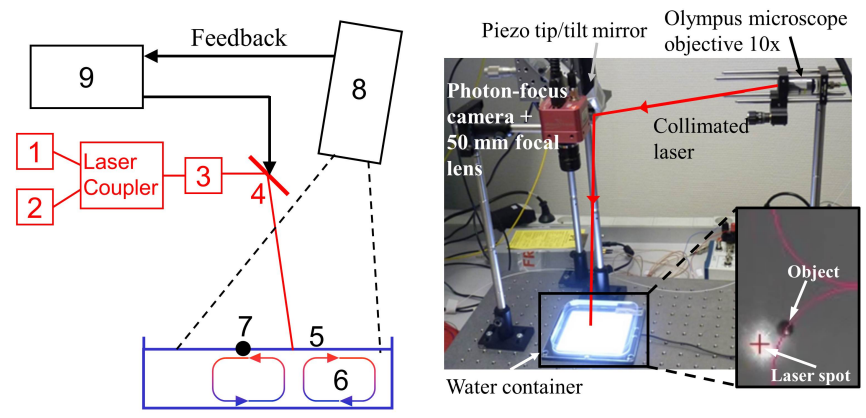

Fig. 1. Experimental setup for thermocapillary convective flow based micromanipulation. An IR laser (1) is coupled with a red laser (2), collimated using a microscope objective (3) and then directed by an actuated mirror (4) towards a point in the water surface (5). The water absorbs the IR laser and so the temperature increases in (5), generating a thermocapillary convective flow (6) which drives a particle (7) along the interface. A camera (8) provides visual feedback, used by a controller (9) which actuates the mirror.

four experiments are performed. The velocity of the particle is presented in Fig. 2(a). When the laser is turned on $(t=0 \mathrm{~s})$, it starts heating the fluid. However, a time delay is needed so that the heat propagates into the fluid and that the flow is established. The particle velocity increases until it reaches a maximum value, between 2 to $6 \mathrm{~mm} / \mathrm{s}$, after around $0.3 \mathrm{~s}$. Then, the velocity decreases as the particle moves away from the laser spot.

The second strategy consists in keeping a constant laser-particle distance $r_{\text {las-part }}$ at each time instant. This is achieved by detecting the particle position in real-time using a vision-based feedback. The laser position is then computed using the particle position and the desired laser-particle distance. However, this actuation technique is not closedloop controlled since the input, which is the laser-particle distance, is kept constant during the whole experiment. Several laser-particle distances are tested: $r_{\text {las-part }}=1.1$, $1.5,1.75,2.0,2.25 \mathrm{~mm}$, each one eight times on four different water samples. Results are given in Fig. 2(b). Regarding the response time, overall, the system attains a quasi-steady state after around $0.8 \mathrm{~s}$. The particle attains faster velocities when it is closer to the laser spot, between 1.9 and $5 \mathrm{~mm} / \mathrm{s}$ at quasi-steady. The standard deviation of velocity at quasi steady-state is in the range of $0.6-1.1 \mathrm{~mm} / \mathrm{s}$.

The two proposed strategies enable to reach velocities of several millimeters per second. As discussed in Sec. IV these performances are highly promising for micromanipulation. The second strategy (laser-particle distance is maintained constant) has the advantage that the particle velocity attains a steady-state value, so it will be adopted in the rest of the paper for controlled manipulation. Thus, a system where the input is the laser-particle distance $r_{\text {las-part }}$ and the output is the particle velocity $\dot{\mathbf{P}}_{\text {part }}$ can be defined. As an example, Fig. 3 presents an open-loop experiment in which the particle is displaced along a straight line.

In all the experiments (Fig. 2(a) and 2(b)), the particle is moving even before the laser is turned on at $t=0 \mathrm{~s}$,

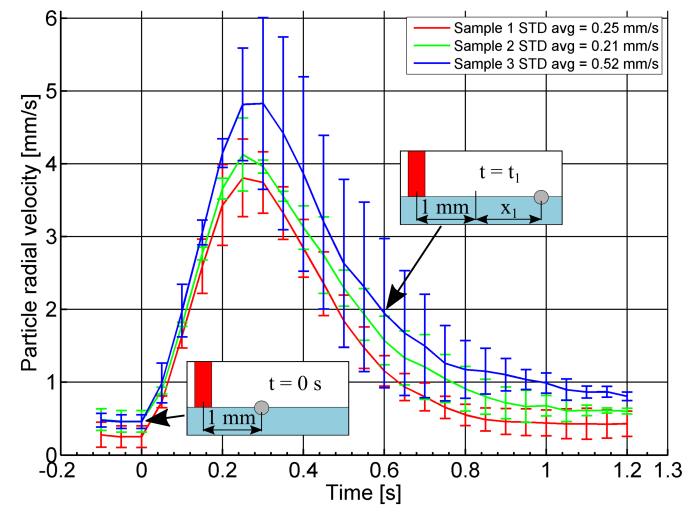

(a) The position of the laser $\mathbf{P}_{\text {las }}$ is fixed. It is set $1 \mathrm{~mm}$ away from the particle position at time $t=0 \mathrm{~s}$. The experiment is performed 4 times on each water sample.

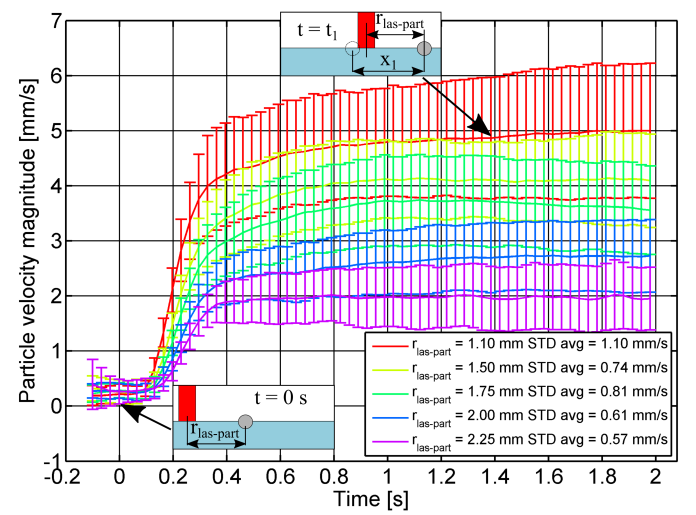

(b) The laser-particle distance $r_{\text {las-part }}$ is kept constant during the whole experiment. Several distances are tested, each one eight times on four different water samples.

Fig. 2. Particle velocity magnitude for different laser inputs.

with a velocity up to $0.9 \mathrm{~mm} / \mathrm{s}$. This is due to fluid movements caused by motion of the air above the liquid surface and slight temperature variations on the liquid surface e.g. cooling from evaporation [24]. The same phenomenon explains why the particle velocity does not decay to zero in the experiments performed (Fig. 2(a)). If the particle has to be maintained at a given position, a closed-loop controller should be implemented in future works to counteract these undesired fluid flows.

In addition, for a given input it can be seen that the experimental results obtained for different trials are similar in trends, but not identical. Precision and repeatability of positioning can thus not be reached with open-loop control. As a first step towards closed-loop control, the rest of this paper proposes a model for the system that could be used in future control laws.

\section{MODEL IDENTIFICATION}

The open-loop experiments presented above highlight the performances of thermocapillary based micromanipulation, but underline the need of closed-loop control to ensure precision and repeatability of actuation. To do so, a model of the system that relates the laser-particle distance $r_{\text {las-part }}$ 


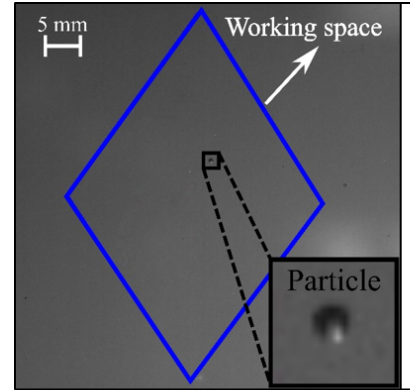

Particle to be manipulated
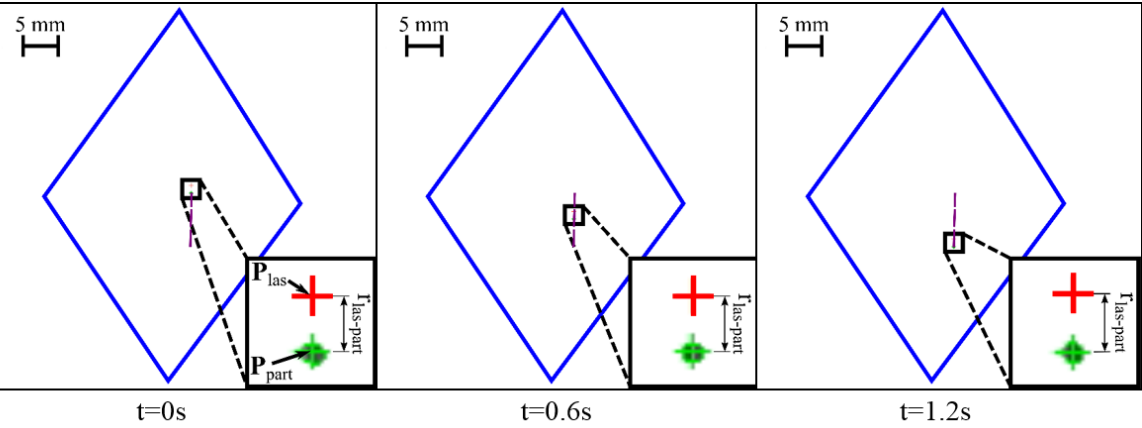

Fig. 3. Example of an open-loop manipulation with a fixed laser-particle distance $r_{\text {las-part }}=1.1 \mathrm{~mm}$. The first image corresponds to the view of the manipulated object (sphere of $500 \mu \mathrm{m}$ of diameter) before manipulation when the white backlight is turned off. In the following images, the backlight is turned on to facilitate the detection of the particle by visual feedback. The particles is displaced along a straight line with a distance of 7.78 mm in $1.2 \mathrm{~s}$.

to the particle velocity $\dot{\mathbf{P}}_{\text {part }}$ is needed. The block diagram representation of this model is given in Fig. 4.

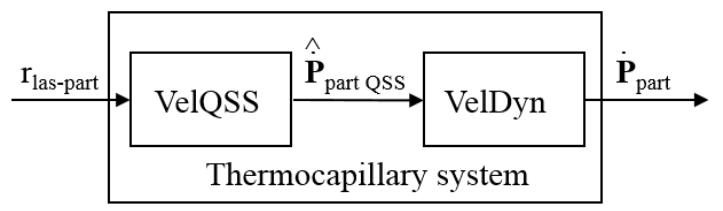

Fig. 4. Block diagram representation of the thermocapillary based micromanipulation system. A laser beam hits the water surface at a certain distance from the particle $r_{\text {las-part }}$. The heat generated by the laser induces flow motion that results in a certain particle velocity $\dot{\mathbf{P}}_{\text {part }}$.

\section{A. Identification of the thermocapillary system}

The goal of this section is to identify the relation between the distance laser-particle $r_{\text {las-part }}$ and the resulting particle velocity $\dot{\mathbf{P}}_{\text {part }}$. This relation is identified based on the step response of the system to a given laser-particle distance $r_{\text {las-part. }}$ Based on Fig. 2(b), a second order model is selected. However, the relation between the attained particle velocity at quasi steady-state, that will be referred as $\dot{\mathbf{P}}_{\text {part QSS }}$ and the laser-particle distance $r_{\text {las-part }}$ is nonlinear. Taking this nonlinearity into consideration, it is proposed to use an estimation of the particle velocity at quasi steady-state $\hat{\mathbf{P}}_{\text {part QSS }}$ as an intermediate variable to define two subsystems as shown in Fig. 4. The first subsystem ("VelQSS" block) is nonlinear and defines $\hat{\mathbf{P}}_{\text {part QSS }}$ in function of the laser-particle distance $r_{\text {las-part }}$. The second subsystem ("VelDyn" block) is a linear system represented by a second order transfer function which is used to model the dynamics of the system.

First, the "VelQSS" block is identified based on the analysis of the particle velocity magnitude at quasi steady-state $\dot{\mathbf{P}}_{\text {part QSS }}$ in function of the input, the laser-particle distance $r_{\text {las-part }}$. Fourteen experiments are performed for laser-particle distances comprised between 1.1 and $4.5 \mathrm{~mm}$. The corresponding particle velocity magnitude at quasi steady-state are given in Fig. 5. An estimation of this velocity $\hat{\dot{P}}_{\text {part }}$ QSS is obtained using an affine regression:

$$
\hat{\dot{\mathbf{P}}}_{\text {part QSS }}=-1.366 r_{\text {las-part }}+5.706
$$

where: $\hat{\dot{\mathbf{P}}}_{\text {part Qss }}$ is expressed in $\left[\mathrm{mm} \cdot \mathrm{s}^{-1}\right]$ and $r_{\text {las-part }}$ is expressed in $[\mathrm{mm}]$. The coefficient of determination for this estimation is $R^{2}=0.897$. Better results could have been obtained using more complex interpolating functions. However since this model will be dedicated to control in future work and should be inverted in real-time, priority is given to simplicity.

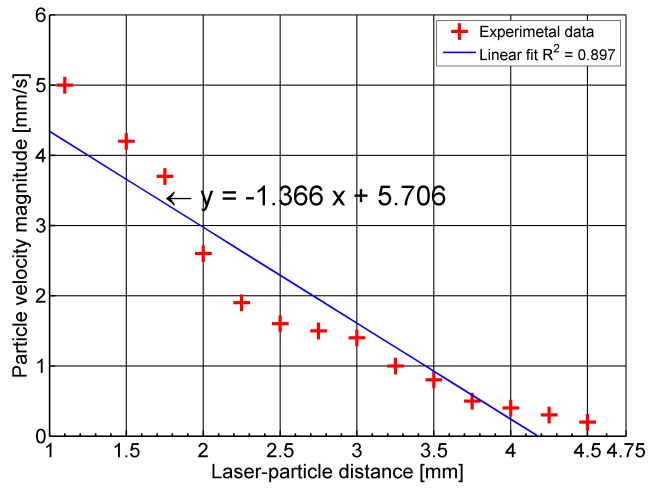

Fig. 5. Measured quasi steady-state particle velocity magnitude $\dot{\mathbf{P}}_{\text {part }}$ QSS in function of the laser-particle distance $r_{\text {las-part }}$ and the corresponding linear fit.

Second, the "VelDyn" block (Fig. 4) is identified based on the dynamics of the particle velocity magnitude $\dot{\mathbf{P}}_{\text {part }}$ in function of the intermediate variable $\dot{\mathbf{P}}_{\text {part QSS }}$, which is computed using Eq. (1). The dynamics of the system is modeled using a second order transfer function with an undamped natural frequency $\omega_{n}$, a damping ratio $\zeta$ and a DC gain of the system $K_{\mathrm{DC}}$. The coefficients $\omega_{n}, \zeta$ and $K_{\mathrm{DC}}$ are obtained from each curve of Fig. 2(b) using Matlab. The identification results, shown in Table I, point out that the coefficients $\omega_{n}, \zeta$ and $K_{\mathrm{DC}}$ do not vary significantly. So it is considered that the mean value of each of them (see the avg. column of Table I) is a good enough representation of the system dynamics'. Using these results, the "VelDyn" block 
is defined by:

$$
\frac{\dot{\mathbf{P}}_{\text {part }}}{\dot{\hat{\mathbf{P}}}_{\text {part QSS }}}=\frac{K_{\mathrm{DC}} \omega_{n}^{2}}{s^{2}+2 \zeta \omega_{n} s+\omega_{n}^{2}}=\frac{63.66}{s^{2}+13.9 s+63.66}
$$

where: $\dot{\mathbf{P}}_{\text {part }}$ and $\hat{\dot{\mathbf{P}}}_{\text {part }}$ Qss are expressed in $\left[\mathrm{mm} \cdot \mathrm{s}^{-1}\right]$.

TABLE I

IDENTIFIED TRANSFER FUNCTION COEFFICIENTS

\begin{tabular}{|c|c|c|c|c|c||c|}
\hline$r_{\text {las-part }}[\mathrm{mm}]$ & 1.1 & 1.5 & 1.75 & 2.0 & 2.25 & avg. \\
\hline$\omega_{n}$ & 9.26 & 7.41 & 7.04 & 8.12 & 7.87 & 7.98 \\
\hline$\zeta$ & 1.05 & 0.86 & 0.82 & 0.96 & 0.67 & 0.87 \\
\hline$K_{\mathrm{DC}}$ & 1.19 & 1.15 & 1.12 & 0.87 & 0.72 & 1.00 \\
\hline
\end{tabular}

\section{B. Discussion on the model}

The system is thus underdamped with a damping coefficient of $\zeta=0.87$. It presents a slight overshoot since the magnitude of the first peak corresponds to $0.4 \%$ of the steady state value. The time to reach and stay within $5 \%$ of the steady state value is around $0.4 \mathrm{~s}$. This time constant is usually lower in common actuation systems (such as dielectrophoresis or magnetophoresis) at the micrometer scale due to the low inertia of the objects. However, the proposed approach requires certain time to onset the flow motion, which increases the time constant. However, this value is still acceptable for micromanipulation.

The identified model is compared to the experimental data. As an example, Fig. 6 shows the comparison between the experimental velocity obtained for a laser-particle distance $r_{\text {las-part }}$ of $1.1 \mathrm{~mm}$ and the proposed model in function of time. The resulting dynamic behavior obtained from the model matches the experimental data with good accuracy. The difference in the estimated particle velocity magnitude is due to the error in the estimation of the particle velocity magnitude at quasi steady-state. However, it can be compensated when closed-loop control will be performed.

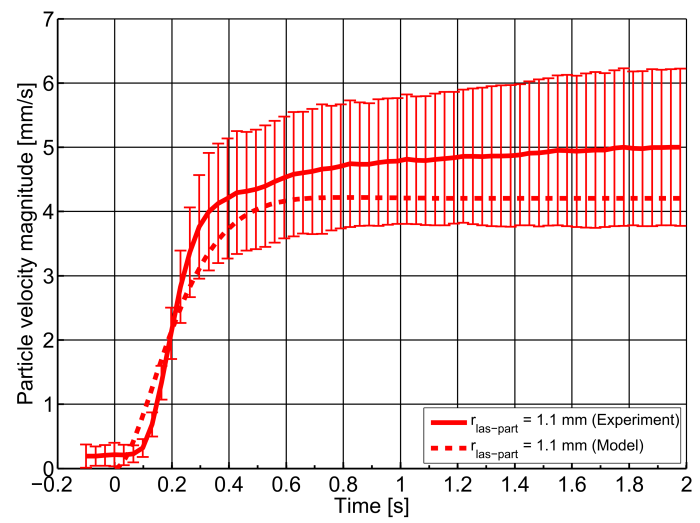

Fig. 6. Particle velocity magnitude $\dot{\mathbf{P}}_{\text {part }}$. Comparison of the characterized model against the experimental data. The solid line corresponds to the mean curve obtained from the experimental data for a laser-particle distance of 1.1 millimeters. The dashed line corresponds to the results obtained using the identified model.

\section{Discussion}

In the Introduction section, non-contact actuation techniques were discussed. Their main characteristics are summarized in Table II. Note that this table presents the usual performances reached by the different micromanipulation techniques. It is out of the scope of the paper to provide a detailed analysis of all state-of-the-art performances attained in specific conditions. In particular, the velocity values or the size of the manipulated objects are given as indications. Better performances can be reached in optimized setups. The size range of the manipulated object is limited here to the micrometric scale, which is the target size range of this paper. Works manipulating larger or smaller objects are not considered here.

Thermocapillary convective flow based micromanipulation achieves fast manipulation velocities (several millimeters per second) on objects of a wide size range, shape and mechanical properties. The condition is that the objects should be hydrophobic to stay at the air/liquid interface. The attainable workspace is large since it is determined by the deflection angle of the mirror and the liquid-mirror distance. Since the system is unstable (the particles are pushed away from the laser source), closed-loop control has to be considered for controlled manipulation. If several objects have to be manipulated independently, strategies similar to the one used in [19] for Marangoni based manipulation could be investigated. Thermocapillary convective flow based micromanipulation is thus a highly promising technique for micromanipulation. The main limitation of the proposed approach is that the objects are manipulated at the air/liquid interface, which restricts the displacements to a plane. However, several applications at micrometer scale, such as conveying [6] or assembly [25] benefit from the planar manipulation. Vortex pumps are also demonstrated in [26] using laser trapping of micro beads placed at an air/liquid interface.

\section{CONCLUSION AND FUTURE WORKS}

In this paper, thermocapillary convective flows are used as an actuation principle for the manipulation of micrometer size particles. An experimental characterization of the system confirms that this approach is promising for micromanipulation. Two actuation strategies are compared, where the laser position is either fixed or maintained at a given distance from the particle. Both strategies enable to move $500 \mu \mathrm{m}$ diameter spherical particles placed at the interface between the air and the liquid with velocities of several millimeters per second. The relevance of the proposed approach compared to magnetophoresis, dielectrophoresis, optical tweezer and Marangoni convection is discussed. Despite its limitation to planar manipulation, thermocapillary based micromanipulation is attractive due to the low requirements on the particle properties, the large workspace and the attained velocities. However, experiments point out that precision and repeatability of positioning are not guaranteed and closedloop control should be considered in future works. 
TABLE II

COMPARISON OF THERMOCAPILLARY BASED MICROMANIPULATION AND OTHER CLASSICAL APPROACHES

\begin{tabular}{|c|c|c|c|c|c|}
\hline & Dielectrophoresis & Magnetophoresis & Optical tweezer & Marangoni manip. & Thermocapillary manip. \\
\hline $\begin{array}{c}\text { Object size } \\
\text { Object property } \\
\text { Workspace / force range } \\
\text { Degrees of freedom } \\
\text { Control of orientation } \\
\text { Control } \\
\text { Velocity } \\
\text { Control of several objects }\end{array}$ & $\begin{array}{c}1-100 \mu \mathrm{m} \\
\text { dielectric } \\
\text { small / small } \\
2.5 \mathrm{D} \\
\text { ok } \\
\text { stable/unstable } \\
\text { several mm/s } \\
\text { possible }\end{array}$ & 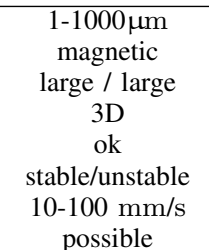 & $\begin{array}{c}1-50 \mu \mathrm{m} \\
\text { transparent } \\
\text { large / very small } \\
3 \mathrm{D} \\
\text { ok } \\
\text { stable } \\
10 \mu \mathrm{m} / \mathrm{s} \\
\mathrm{ok}\end{array}$ & $\begin{array}{c}\text { 1-1000 } \mu \mathrm{m} \\
\text { hydrophilic } \\
\text { large / medium } \\
2 \mathrm{D} \\
\text { no } \\
\text { stable } \\
\text { several mm/s } \\
\text { possible }\end{array}$ & $\begin{array}{c}1-1000 \mu \mathrm{m} \\
\text { hydrophobic } \\
\text { large / medium } \\
2 \mathrm{D} \\
\text { no } \\
\text { unstable } \\
\text { several mm/s } \\
\text { to be done }\end{array}$ \\
\hline
\end{tabular}

A model of the system is identified based on the experimental results. Even if the numerical values provided in the paper are related to the setup here used, the methodology remains general so that it can be applied to other systems using thermocapillary convective flows for particle manipulation. This model is a key step towards closed-loop control, and it is used in [27] for controlled thermocapillary based micromanipulation.

\section{ACKNOWLEDGMENT}

This research has been funded by the Interuniversity Attraction Poles Programme (IAP 7/38 MicroMAST) initiated by the Belgian Science Policy Office, by the Labex ACTION project (contract "ANR-11-LABX-01-01") and by the Conseil Régional de Franche-Comté.

\section{REFERENCES}

[1] R. Pethig, "Review Article Dielectrophoresis: Status of the theory, technology, and applications," Biomicrofluidics, vol. 4, no. 2, June 2010.

[2] M. Kharboutly and M. Gauthier, "High speed closed loop control of a dielectrophoresis-based system," in IEEE International Conference on Robotics and Automation, 2013, pp. 1446-1451.

[3] J. Zemanek, T. Michalek, and Z. Hurak, "Feedback control for noiseaided parallel micromanipulation of several particles using dielectrophoresis," Electrophoresis, vol. 36, no. 13, pp. 1451-1458, 2015.

[4] I. Khalil, K. Youakim, A. Sanchez, and S. Misra, "Magnetic-based motion control of sperm-shaped microrobots using weak oscillating magnetic fields," in IEEE/RSJ International Conference on Intelligent Robots and Systems, Sept. 2014, pp. 4686-4691.

[5] P. Ryan and E. Diller, "Five-degree-of-freedom magnetic control of micro-robots using rotating permanent magnets," in IEEE International Conference on Robotics and Automation, 2016, pp. 1731-1736.

[6] M. Dkhil, M. Kharboutly, A. Bolopion, S. Régnier, and M. Gauthier, "Closed loop control of a magnetic particle at the air/liquid interface," IEEE Transactions on Automation Science and Engineering, pp. 1-13, jun 2015.

[7] D. de Lanauze, O. Felfoul, J.-P. Turcot, M. Mohammadi, and S. Martel, "Three-dimensional remote aggregation and steering of magnetotactic bacteria microrobots for drug delivery applications," The International Journal of Robotics Research, vol. 33, no. 3, pp. 359-374, 2014.

[8] S. Bouchebout, A. Bolopion, J. Abrahamians, and S. Régnier, "An overview of multiple dof magnetic actuated micro-robots," Journal of Micro-Nano Mechatronics, vol. 7, no. 4, pp. 97-113, 2012.

[9] A. Oulmas, S. Régnier, and N. Andreff, "Closed-loop 3d path following of scaled-up helical microswimmers," in IEEE International Conference on Robotics and Automation, 2016, pp. 1725-1730.

[10] E. Diller, J. Giltinan, and M. Sitti, "Independent control of multiple magnetic microrobots in three dimensions," The International Journal of Robotics Research, vol. 32, no. 5, pp. 614-631, 2013.

[11] F. Ullrich, C. Bergeles, J. Pokki, O. Ergeneman, S. Erni, G. Chatzipirpiridis, S. Pane, C. Framme, and B. J. Nelson, "Mobility Experiments With Microrobots for Minimally Invasive Intraocular Surgery," Investigative Ophthalmology \& Visual Science, vol. 54, no. 4, pp. 28532863, 2013.
[12] C. Pacoret, R. Bowman, G. Gibson, S. Haliyo, D. Carberry, A. Bergander, S. Régnier, and M. Padgett, "Touching the microworld with forcefeedback optical tweezers," Optics express, vol. 17, no. 12, pp. 10259 $10264,2009$.

[13] K. Onda and F. Arai, "Parallel teleoperation of holographic optical tweezers using multi-touch user interface," in IEEE International Conference on Robotics and Automation, May 2012, pp. 1069 -1074.

[14] E. Avci and G. Z. Yang, "Development of a microhand using direct laser writing for indirect optical manipulation," in IEEE/RSJ International Conference on Intelligent Robots and Systems, 2016, pp. 51255130.

[15] H. Yang, X. Gou, Y. Wang, T. M. Fahmy, A. Y.-H. Leung, J. Lu, and D. Sun, "A dynamic model of chemoattractant-induced cell migration," Biophysical journal, vol. 108, no. 7, pp. 1645-1651, 2015.

[16] E. Diller, N. Zhang, and M. Sitti, "Bonding methods for modular micro-robotic assemblies," in IEEE International Conference on Robotics and Automation, 2013, pp. 2588-2593.

[17] K. Schutze, H. Posl, and G. Lahr, "Laser micromanipulation systems as universal tools in cellular and molecular biology and in medicine," Cellular and Molecular Biology, vol. 44, no. 5, pp. 735-746, 1998.

[18] C. Elbuken, L. Gui, C. L. Ren, M. Yavuz, and M. B. Khamesee, "Design and analysis of a polymeric photo-thermal microactuator," Sensors and Actuators A: Physical, vol. 147, no. 1, pp. 292-299, Sept. 2008.

[19] E. M. Munoz, J. E. Quispe, and E. Vela, "Closed-loop selective manipulation of multiple microparticles by controlling the transient regime of Marangoni flows," in IEEE/RSJ International Conference on Intelligent Robots and Systems, Oct. 2016, pp. 5137-5142.

[20] M. F. Schatz and G. P. Neitzel, "Experiments on thermocapillary instabilities," Annual Review of Fluid Mechanics, vol. 33, no. 1, pp. 93-127, 2001.

[21] R. Terrazas Mallea, A. Bolopion, J.-C. Beugnot, M. Gauthier, and P. Lambert, "Laser-induced thermocapillary convective flows: A new approach for non-contact actuation at microscale at the fluid/gas interface," IEEE/ASME Transactions on Mechatronics, vol. PP, no. 99, pp. $1-11,2016$.

[22] K. Ishii, W. Hu, and A. Ohta, "Cooperative micromanipulation using optically controlled bubble microrobots," in IEEE International Conference on Robotics and Automation, 2012, pp. 3443-3448.

[23] W. Hu, Q. Fan, and A. T. Ohta, "Interactive actuation of multiple opto-thermocapillary flow-addressed bubble microrobots," Robotics and Biomimetics, vol. 1, no. 1, pp. 1-6, 2014.

[24] J. P. Longtin, K. Hijikata, and K. Ogawa, "Laser-induced surfacetension-driven flows in liquids," International Journal of Heat and Mass Transfer, vol. 42, no. 1, pp. 85 - 93, 1999.

[25] J. M. Ng, M. J. Fuerstman, B. A. Grzybowski, H. A. Stone, and G. M. Whitesides, "Self-assembly of gears at a fluid/air interface," Journal of the American Chemical Society, vol. 125, no. 26, pp. 7948-7958, 2003.

[26] A. Jesacher, S. Furhapter, C. Maurer, S. Bernet, and M. Ritsch-Marte, "Holographic optical tweezers for object manipulations at an air-liquid surface," Optics express, vol. 14, no. 13, pp. 6342-6352, 2006.

[27] R. Terrazas Mallea, A. Bolopion, J.-C. Beugnot, M. Gauthier, and P. Lambert, "Closed-loop particle positioning control using laserinduced thermocapillary convective flows at the fluid/gas interface at micrometric scale," submitted in IEEE/ASME Transactions on Mechatronics. 